OPEN ACCESS

Edited by:

Helen Zgurskaya,

The University of Oklahoma,

United States

Reviewed by:

Hiroyuki Yamada,

Japan Anti Tuberculosis Association,

Japan

Jon William Weeks,

United States Food and Drug

Administration, United States

${ }^{*}$ Correspondence:

Xiaoke Hao

haoxkg@fmmu.edu.cn

Valeri N. Danilenko

valerid@vigg.ru

Ying Zhang

yzhang@jhsph.edu

Specialty section: This article was submitted to Antimicrobials, Resistance and Chemotherapy,

a section of the journal

Frontiers in Microbiology

Received: 04 December 2018

Accepted: 25 January 2019

Published: 19 February 2019

Citation:

Liu J, Shi W, Zhang S, Hao X, Maslov DA, Shur KV, Bekker OB, Danilenko VN and Zhang Y (2019) Mutations in Efflux Pump Rv1258c

(Tap) Cause Resistance

to Pyrazinamide, Isoniazid, and Streptomycin in M. tuberculosis.

Front. Microbiol. 10:216.

doi: 10.3389/fmicb.2019.00216

\section{Mutations in Efflux Pump Rv1258c (Tap) Cause Resistance to Pyrazinamide, Isoniazid, and Streptomycin in $M$. tuberculosis}

\author{
Jiayun Liu',2, Wanliang Shi', Shuo Zhang'2, Xiaoke Hao ${ }^{1 *}$, Dmitry A. Maslov, \\ Kirill V. Shur ${ }^{3}$, Olga B. Bekker ${ }^{3}$, Valery N. Danilenko ${ }^{3 *}$ and Ying Zhang ${ }^{2 *}$
}

\footnotetext{
' Department of Clinical Laboratory, Xijing Hospital, Fourth Military Medical University, Xi'an, China, ${ }^{2}$ Department of Molecular Microbiology and Immunology, Bloomberg School of Public Health, Johns Hopkins University, Baltimore, MD, United States, ${ }^{3}$ Laboratory of Bacterial Genetics, Vavilov Institute of General Genetics, Russian Academy of Sciences, Moscow, Russia
}

Although drug resistance in Mycobacterium tuberculosis is mainly caused by mutations in drug activating enzymes or drug targets, there is increasing interest in the possible role of efflux in causing drug resistance. Previously, efflux genes have been shown to be upregulated upon drug exposure or implicated in drug resistance in overexpression studies, but the role of mutations in efflux pumps identified in clinical isolates in causing drug resistance is unknown. Here we investigated the role of mutations in efflux pump Rv1258c (Tap) from clinical isolates in causing drug resistance in M. tuberculosis. We constructed point mutations V219A and S292L in Rv1258c in the chromosome of $M$. tuberculosis and the point mutations were confirmed by DNA sequencing. The susceptibility of the constructed $M$. tuberculosis Rv1258c mutants to different tuberculosis drugs was assessed using conventional drug susceptibility testing in $7 \mathrm{H} 11$ agar in the presence and absence of efflux pump inhibitor piperine. A C14-labeled PZA uptake experiment was performed to demonstrate higher efflux activity in the M. tuberculosis Rv1258c mutants. Interestingly, the V219A and S292L point mutations caused clinically relevant drug resistance to pyrazinamide (PZA), isoniazid (INH), and streptomycin (SM), but not to other drugs in M. tuberculosis. While V219A point mutation conferred low-level drug resistance, the S292L mutation caused a higher level of resistance. Efflux inhibitor piperine inhibited INH and PZA resistance in the S292L mutant but not in the V219A mutant. The S292L mutant had higher efflux activity for pyrazinoic acid (the active form of PZA) than the parent strain. We conclude that point mutations in the efflux pump Rv1258c in clinical isolates can confer clinically relevant drug resistance, including PZA resistance, and could explain some previously unaccounted drug resistance in clinical strains. Future studies need to take efflux mutations into consideration for improved detection of drug resistance in $M$. tuberculosis and address their role in affecting treatment outcome in vivo.

Keywords: drug resistance, efflux pump, mutations, tuberculosis, pyrazinamide 


\section{INTRODUCTION}

Multidrug resistant (MDR) tuberculosis (MDR-TB), defined by resistance to at least the two frontline drugs isoniazid (INH) and rifampin (RIF), poses a significant challenge to effective treatment and control of the disease. In 2015, there were at least 480,000 cases of MDR-TB and about $9.5 \%$ of MDR-TB cases were extensively drug resistant TB, or XDR-TB (WHO, 2018). Drug resistance in the causative agent Mycobacterium tuberculosis is mainly caused by mutations affecting enzymes involved in drug activation or by alterations or overexpression of drug targets (Zhang and Yew, 2009). However, efflux pumps, which have been found to cause antibiotic resistance in many other bacteria ( $\mathrm{Li}$ and Nikaido, 2009) and also non-tuberculous mycobacteria (Liu et al., 1996; Ainsa et al., 1998) have only recently been shown to be involved in clinical drug resistance in $M$. tuberculosis in the case of clofazimine and bedaquiline (Andries et al., 2014; Hartkoorn et al., 2014).

The efflux protein Rv1258c, also called Tap or P55, was previously shown to be involved in resistance to different drugs in overexpression studies involving Mycobacterium megmatis and Mycobacterium bovis BCG (Ramon-Garcia et al., 2012; Shur et al., 2017). For example, overexpression of Rv1258c was shown to confer resistance to INH, RIF, ethambutol, PAS, and ethambutol in M. bovis BCG (Ramon-Garcia et al., 2012). Additionally, Jiang et al. (2008) showed that Rv1258c was overexpressed upon exposure to INH and RIF in MDRTB clinical isolates and hypothesized that its overexpression may cause drug resistance in clinical TB strains. Furthermore, various single nucleotide polymorphisms (SNPs) in different efflux pump genes Rv0194, Rv1217, Rv1258c, Rv1273, Rv1877, Rv1250, and Rv2688 in XDR-TB clinical isolates have recently been identified (Kanji et al., 2017). However, the relevance of these SNPs in causing drug resistance in clinical strains has been unclear. In this study, we identified SNPs in the efflux gene $r v 1258 c$ (tap) from clinical isolates of $M$. tuberculosis and then evaluated the significance of the SNPs in causing clinically relevant drug resistance by introducing these point mutations into the genome of the isogenic strain of $M$. tuberculosis. We demonstrate that mutations (V219A, S292L) in efflux pump Tap Rv1258c identified in clinical strains can cause clinically relevant drug resistance in $M$. tuberculosis.

\section{MATERIALS AND METHODS}

\section{Bacterial Strains, Plasmids and Drugs}

Mycobacterium tuberculosis $\mathrm{H} 37 \mathrm{Ra}$ was grown at $37^{\circ} \mathrm{C}$ in Middlebrook $7 \mathrm{H} 9$ liquid medium or on $7 \mathrm{H} 11$ agar plates supplemented with $10 \% \quad(\mathrm{v} / \mathrm{v})$ albumin-dextrose-catalase (ADC, Becton Dickinson, Sparks, MD, United States) plus $0.5 \%(\mathrm{v} / \mathrm{v})$ glycerol, $0.25 \%(\mathrm{v} / \mathrm{v})$ Tween 80 . Plasmids p2NIL and pGOAL19 used in this study were obtained from Addgene (Cambridge, MA, United States). isoniazid (INH), rifampicin (RIF), streptomycin (SM), ethambutol, pyrazinamide (PZA), levofloxacin, amikacin, cycloserine, p-aminosalicylic acid (PAS), clofazimine (CFZ), tetracycline, linezolid, clarithromycin, and piperine were purchased from Sigma-Aldrich (St Louis, MO, United States). The drugs were dissolved in DMSO and further diluted to obtain the desired concentrations in culture media for drug susceptibility testing (see below).

\section{Construction of Rv1258c Point Mutation Mutants by Homologs Recombination}

The Rv1258c mutants were constructed in M. tuberculosis H37Ra by homologous recombination as described previously (Parish and Stoker, 2000). The mutant construction and drug susceptibility testing were performed in BSL2 laboratory following institutional biosafety procedures. The reason we used the avirulent $M$. tuberculosis H37Ra strain is because it is a good surrogate of its virulent parent strain $\mathrm{H} 37 \mathrm{Rv}$ in terms of drug susceptibility profiles (Heinrichs et al., 2018). Briefly, the Rv1258c gene was amplified with its adjacent $1 \mathrm{~kb}$ fragment on both sides from $M$. tuberculosis genomic DNA and cloned into p2NIL plasmid vector. Then, the mutated constructs Rv1258c V219A and S292L were obtained by QuikChange II XL site-directed mutagenesis kit (Agilent, Santa Clara, CA, United States) with primers TPV219AF: $\quad 5$ 'TTCGCCTGGAACCTGCGGGTATTGCG CACCC-3', TPV219AR: 5'-CCAGGCGAAGCGCAGCCCCTCG GCGATCCC-3', TPS292LR: 5'-CCCCGTCGCGTGACCAT GCTGACCGCGGTTCTTACCCTGGG-3', and TPS292LR: 5'-CCCAGGGTAAGAACCGCGGTCAGCATGGTCACGCGAC GGGG-3'. pGOAL19 cassette was cloned into the PacI site of p2NIL containing the mutant (V219A and S292L) version of the Rv1258c gene to form suicide delivery constructs. The suicide delivery plasmid DNA was subjected to $100 \mathrm{~mJ} / \mathrm{cm} 2$ of UV irradiation followed by addition of $200 \mu \mathrm{l}$ of electrocompetent mycobacteria. Electroporation was performed with the parameters $2.5 \mathrm{kV}, 1000 \Omega, 25 \mathrm{mF}$. The electroporated cells were added with $200 \mu \mathrm{l}$ 7H9-Tween 80 recovery medium and incubated at $37^{\circ} \mathrm{C}$ for $24 \mathrm{~h}$ followed by plating onto $7 \mathrm{H} 11 / \mathrm{ADC}$ plates containing kanamycin at $20 \mu \mathrm{g} / \mathrm{ml}$, hygromycin at $50 \mu \mathrm{g} / \mathrm{ml}$, and X-Gal at $50 \mu \mathrm{g} / \mathrm{ml}$. The plates were incubated at $37^{\circ} \mathrm{C}$ for $4-5$ weeks until colonies appeared. Single crossover transformants were picked and re-streaked onto fresh $7 \mathrm{H} 11 / \mathrm{ADC}$ without any antibiotics and incubated at $37^{\circ} \mathrm{C}$ for 2-3 weeks. A loopful of bacteria from the plates was resuspended in $1 \mathrm{ml}$ of $1-\mathrm{mm}$ glass beads and $3 \mathrm{~mL}$ of 7H9/ADC/Tween 80 and vortexed vigorously. Serial dilutions were plated onto plates containing X-gal with and without sucrose at $2 \%(\mathrm{w} / \mathrm{v})$ and incubated for 4 weeks until the potential double cross-over transformants appeared. The kanamycin-sensitive colonies were screened using colony-PCR followed by DNA sequencing to confirm the desired point mutation being constructed in the genome of M. tuberculosis H37Ra.

\section{Drug Susceptibility Testing and Isoniazid/Piperine Combination Studies}

The Rv1258c V219A and S292L mutants and parent strain M. tuberculosis $\mathrm{H} 37 \mathrm{Ra}$ were grown in $7 \mathrm{H} 9 / \mathrm{ADC} /$ Tween 80 
medium at $37^{\circ} \mathrm{C}$ for $2-3$ weeks [approximately $1 \times 10^{8}$ colonyforming units $(\mathrm{CFU}) / \mathrm{ml}$ ] when they were diluted in serial 10 fold dilutions and inoculated onto $7 \mathrm{H} 11$ agar plates containing different concentrations of drugs in triplicate. The following drug concentrations were used: $\operatorname{SM}(0.5,1,2,4$, and $8 \mu \mathrm{g} / \mathrm{ml})$. INH $(0.125,0.25,0.5,1,2,4,8,16,32$, and $64 \mu \mathrm{g} / \mathrm{ml})$, PZA (50, 100, 200, 400, 800, and $1600 \mu \mathrm{g} / \mathrm{ml}, \mathrm{pH} 6.0)$, PAS $(0.25,0.5,1,2,4,8,16,32,64$, and $128 \mu \mathrm{g} / \mathrm{ml})$, RIF $(0.25,0.5,1,2$, and $4 \mu \mathrm{g} / \mathrm{ml})$, ethambutol $(1,2,4,8$, and $16 \mu \mathrm{g} / \mathrm{ml})$, levofloxacin $(0.25,0.5,1,2$, and $4 \mu \mathrm{g} / \mathrm{ml})$, amikacin $(0.5,1,2,4$, and $8 \mu \mathrm{g} / \mathrm{ml})$, cycloserine $(5,10,20,40$, and $80 \mu \mathrm{g} / \mathrm{ml}), \operatorname{CFZ}(0.125,0.25,0.5,1$, and $2 \mu \mathrm{g} / \mathrm{ml})$, tetracycline $(0.5,1,2,4,8,16,32,64$, and $128 \mu \mathrm{g} / \mathrm{ml})$, linezolid $(0.25$, $0.5,1,2$, and $4 \mu \mathrm{g} / \mathrm{ml})$, and clarithromycin $(0.125,0.25$, 0.5 , and $1,2 \mu \mathrm{g} / \mathrm{ml}$ ). The plates were incubated at $37^{\circ} \mathrm{C}$ in $5 \% \mathrm{CO}_{2}$ for $3-4$ weeks. The $\mathrm{INH} /$ piperine combination study was performed on the Rv1258c V219A and S292L mutants with different concentrations of INH tested in the presence of increasing concentrations of piperine $(0,5,10$, 20 , and $40 \mu \mathrm{g} / \mathrm{ml})$.

\section{PZA Uptake and Accumulation Experiments}

Two-week-old cultures of Rv1258c V219A and S292L mutants of M. tuberculosis H37Ra, grown in in7H9/ADC/Tween 80 medium, were harvested and the cell pellets were resuspended in $7 \mathrm{H} 9$ medium $(\mathrm{pH} 6.6)$ at $5 \times 10^{8}$ cells $/ \mathrm{ml}$. The PZA uptake and accumulation study was performed as described previously (Zhang et al., 1999). Briefly, $\left[{ }^{14} \mathrm{C}\right] \mathrm{PZA}$, purchased from Vitrax (Placentia, CA, United States), was added to the cell suspensions to a concentration of $1 \mu \mathrm{Ci} / \mathrm{ml}$ and the cell mixtures were incubated at $37^{\circ} \mathrm{C}$. At different time points, $50 \mu \mathrm{l}$ portions were removed and washed with

TABLE 1 | Susceptibility of M. tuberculosis efflux pump Rv1258c point mutation mutants to different TB drugs.

\begin{tabular}{|c|c|c|c|}
\hline \multirow[t]{2}{*}{ Drugs } & \multirow{2}{*}{$\begin{array}{c}\begin{array}{c}\text { MIC } \\
\text { breakpoints }\end{array} \\
\begin{array}{c}\text { H37Ra } \\
(\mu \mathrm{g} / \mathrm{ml})\end{array}\end{array}$} & \multicolumn{2}{|c|}{ Drug susceptibility results\# } \\
\hline & & Rv1258c V219A & Rv1258c S292L \\
\hline Isoniazid & 0.05 & $\mathrm{R}(\mathrm{MIC} 0.125 \mu \mathrm{g} / \mathrm{ml})$ & R (MIC $32 \mu \mathrm{g} / \mathrm{ml})$ \\
\hline Pyrazinamide & 50 & R (MIC $100 \mu \mathrm{g} / \mathrm{ml})$ & $\mathrm{R}(\mathrm{MIC} 800 \mu \mathrm{g} / \mathrm{ml})$ \\
\hline Streptomycin & 0.5 & S & $\mathrm{R}(\mathrm{MIC} 4.0 \mu \mathrm{g} / \mathrm{ml})$ \\
\hline Rifampin & 1.0 & S & $S$ \\
\hline Ethambutol & 5.0 & S & S \\
\hline Amikacin & 1.0 & S & S \\
\hline Cycloserine & 10 & S & S \\
\hline $\begin{array}{l}\text { p-aminosalicylic } \\
\text { acid (PAS) }\end{array}$ & 0.20 & S & S \\
\hline Clofazimine & 0.20 & S & S \\
\hline Tetracycline & 8.0 & S & S \\
\hline Linezolid & 0.5 & S & S \\
\hline Clarithromycin & 2.0 & s & s \\
\hline
\end{tabular}

\# S indicates "susceptible"; R indicates "resistant".
$1 \times$ PBS buffer (pH 6.6) with $0.1 \mathrm{M} \mathrm{LiCl}$ by filtration on $0.45 \mu \mathrm{m}$-pore-size nitrocellulose filters by using a vacuum pump. The amount of radioactivity associated with the

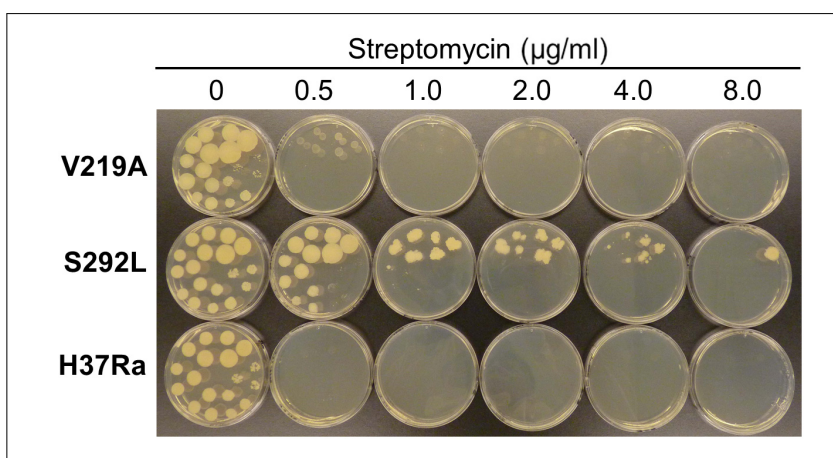

FIGURE 1 | The Rv1258c S292L mutant of Mycobacterium tuberculosis has higher level of resistance to streptomycin (SM) than the V219 mutant.

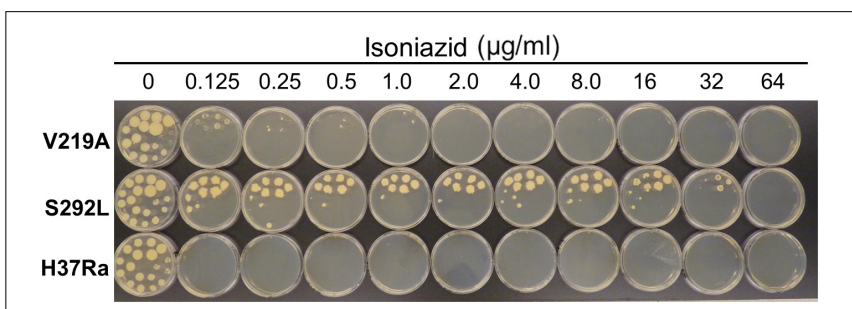

FIGURE 2 | The Rv1258c S292L mutant of M. tuberculosis is highly resistant to isoniazid (INH), while the V219A mutant is only slightly resistant.

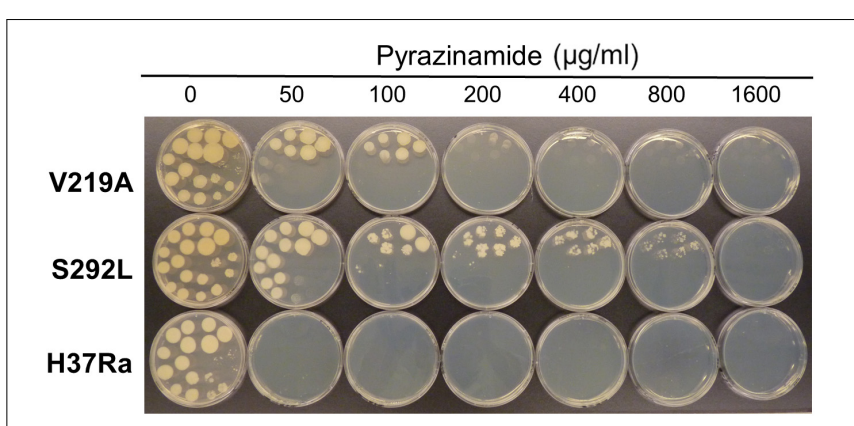

FIGURE 3 | The Rv1258c S292L mutant of M. tuberculosis has a higher level of PZA resistance than the V219A mutant.

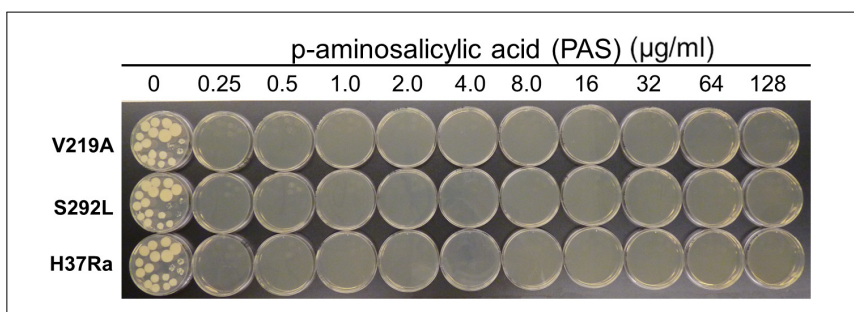

FIGURE 4 | The Rv1258c S292L and V219A mutants of M. tuberculosis remain susceptible to PAS. 
bacterial cells was determined by autoradiography and scintillation counting.

\section{RESULTS AND DISCUSSION}

\section{Identification of Point Mutations in Rv1258c From Clinical Isolates of \\ M. tuberculosis and Construction of Rv1258c Point Mutations in}

\section{M. tuberculosis}

To determine possible roles of efflux pumps in causing drug resistance, we performed a database search for mutations in the efflux pump Rv1258c (Tap) among clinical isolates whose whole genome sequences are deposited in the GenBank NCBI database. Several single nucleotide polymorphisms were found in the efflux gene Rv1258c (D23V, V219A, and S292L) in different clinical isolates of M. tuberculosis, which may affect the function of the efflux protein. This is consistent with the identification of Rv1258c P369T and G391R in XDR-TB clinical isolates from sequenced genomes of $M$. tuberculosis strains from Pakistan (Kanji et al., 2017).

To address the role of the identified point mutations in Rv1258c, we attempted to construct the point mutations D23V, V219A, and S292L by site-directed mutagenesis by PCR and the altered mutant sequences were confirmed to be correct by DNA sequencing. We were able to successfully construct Rv1258c point mutations V219A and S292L, but not D23V (for unknown reasons) in M. tuberculosis H37Ra. It is worth noting that the constructed Rv1258c point mutation V219A and S292L mutant strains had no growth defect or altered morphology as seen in Rv1258c deletion mutant strain (Ramon-Garcia et al., 2012). Therefore, we evaluated the susceptibility of Rv1258c point mutation V219A and S292L mutants to various drugs as described below.
A
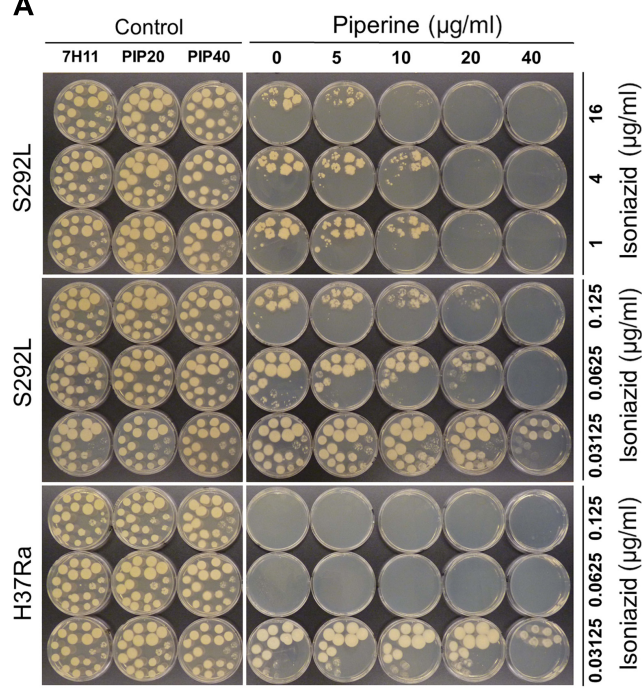

C

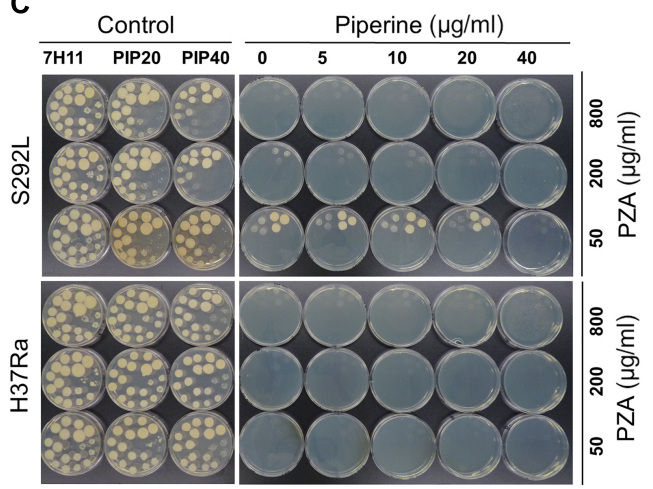

B

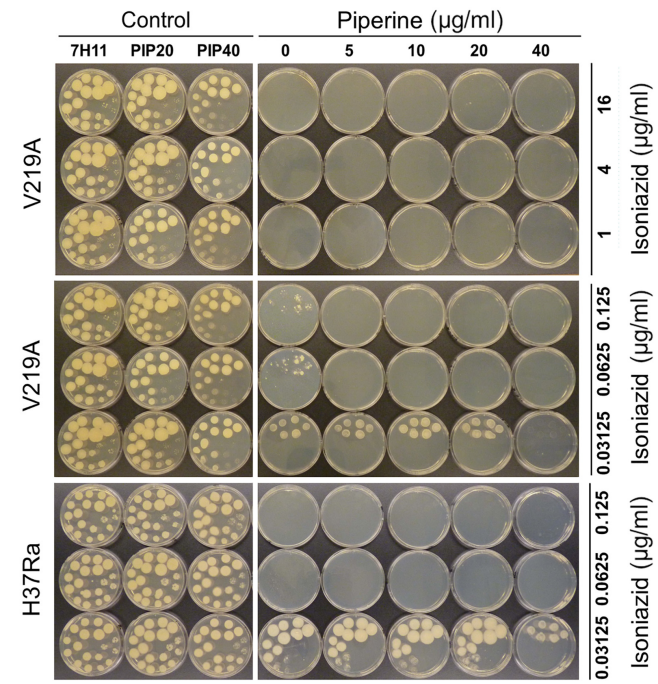

FIGURE 5 | Effect of efflux pump inhibitor Piperine on INH resistance and PZA resistance in Rv1258c mutants of $M$. tuberculosis. Piperine at $40 \mathrm{mg} / \mathrm{ml}$ but at lower concentrations inhibited or reduced the level of INH resistance (A) and PZA resistance (C) in the Rv1258c S292L mutant, but not in the V219A mutant (B). 


\section{Rv1258c Point Mutations Confer Resistance to PZA, INH, and SM}

The results of drug susceptibility testing showed that Rv1258c S292L mutant was more resistant to SM, INH, and PZA than the parent strain H37Ra (Table 1). However, Rv1258c V219A point mutation caused a lower level of resistance to the above drugs than the S292L mutation. It is noteworthy that while V219A mutation only caused a marginal level of INH resistance $(\mathrm{MIC}=0.125 \mu \mathrm{g} / \mathrm{ml})$, S292L mutation caused a very high level of INH resistance $($ MIC $=32 \mu \mathrm{g} / \mathrm{ml}$ ) (Table 1). This latter finding is significant as it offers a possible alternative mechanism of INH resistance in addition to mutations in katG (Zhang et al., 1992; Zhang and Yew, 2009) or inhA (Banerjee et al., 1994). Introduction of the V219A and S292L mutations in $M$. tuberculosis did not alter the susceptibility to other drugs including RIF, ethambutol, levofloxacin, amikacin, cycloserine, PAS, CFZ, tetracycline, linezolid and clarithromycin (Figures 1-4 and Table 1). Our finding that Rv1258c S292L point mutation conferred resistance to SM and INH is consistent with the previous observation of overexpression of Rv1258c (Ramon-Garcia et al., 2012). However, the Rv1258c S292L and V219A mutations did not alter susceptibility to other drugs such as tetracycline, RIF, EMB, CFZ, while previous studies have demonstrated that overexpression of Rv1258c could cause resistance to these drugs (Ainsa et al., 1998; Ramon-Garcia et al., $2009,2012)$. One possibility for such a discrepancy is that the point mutations at S292L and V219A cause differential binding to different drugs such that they have differential effects on causing selective drug resistance to some but not other drugs, as seen in overexpression study of the wild-type functional protein Rv1258c (Ramon-Garcia et al., 2009, 2012).

Previous studies that evaluated drug resistance conferred by overexpression of Rv1258c did not test PZA, presumably because of the well-known problem with its susceptibility testing (Zhang and Mitchison, 2003). Here, it is of interest to note that introducing the S292L point mutation in Rv1258c into the chromosome of $M$. tuberculosis conferred a high level of PZA resistance $(800 \mu \mathrm{g} / \mathrm{ml})$, whereas the V219A mutation caused a low level of PZA resistance $(100-200 \mu \mathrm{g} / \mathrm{ml})$ (Figure 3). Although PZA resistance is mostly caused by $p n c A$ mutations (Zhang Y. et al., 2013) and less commonly by rpsA (Shi et al., 2011), panD (Zhang S. et al., 2013), and clpC1 (Yee et al., 2017; Zhang S. et al., 2017) mutations, some PZA-resistant M. tuberculosis strains without mutations in the above known genes do exist (Cheng et al., 2000) (Zhang Y, unpublished). Recently, we have shown that overexpression of efflux proteins Rv0191, Rv3756c, Rv3008, and Rv1667c could all confer lowlevel PZA resistance in M. tuberculosis (Zhang Y. et al., 2017), indicating a role of efflux in PZA resistance. However, not all efflux pumps are involved in PZA resistance as overexpression of efflux pump DrrAB in M. tuberculosis did not confer PZA resistance (Zhang Y, unpublished). Here it is worth noting that by constructing point mutations in the genome of the efflux pump gene $r v 1258 c$, we were able to convincingly demonstrate that the S292L mutation is indeed causative of PZA resistance. This finding suggests that mutations in Rv1258c could be a potential new mechanism of PZA resistance in clinical isolates without known structural gene ( $p n c A, r p s A$, panD) mutations. Future studies are needed to determine how frequently such mechanisms of PZA resistance mediated by mutations in Rv1258c occur in clinical isolates. Although we were able to demonstrate that the point mutations in Rv1258c confer drug resistance in M. tuberculosis, it remains to be determined if the mutations caused the elevated drug resistance phenotype through affecting the stability (Zhu et al., 2011), expression levels (Kardan-Yamchi et al., 2019), or drug affinity (Cloete et al., 2018) of the mutant Rv1258c protein. Future studies are needed to address these possibilities.

\section{Piperine Inhibits the INH and PZA Resistance in the Rv1258c S292L Mutant}

Piperine is a known inhibitor of efflux pump protein Rv1258c in M. tuberculosis (Sharma et al., 2010). To determine if the S292L point mutation mediated higher level of resistance is due to elevated efflux activity of the Rv1258c S292L mutant protein, we tested if piperine could antagonize the INH and PZA resistance mediated by the Rv1258c S292L mutant protein. The piperine/INH or piperine/PZA combination study showed that piperine reduced both INH and PZA resistance or increased INH and PZA susceptibility in the Rv1258c S292L mutant M. tuberculosis strain (Figures $\mathbf{5 A}, \mathbf{C}$ ), but much less so in the

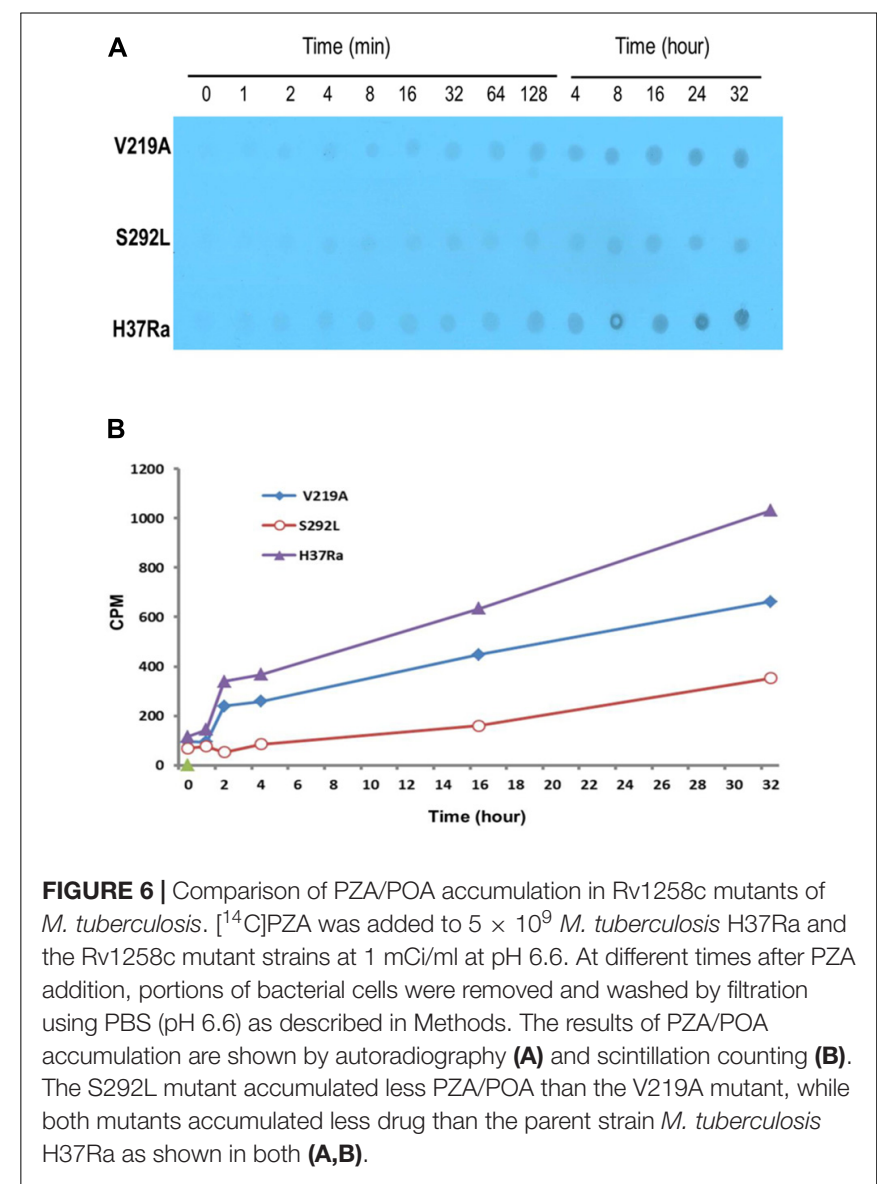


V219A mutant strain, presumably due to the much weaker efflux activity of the V219A mutant (Figure 5B) compared with the S292L mutant. The inhibitory effect of piperine on reducing INH resistance in the V219A mutant could only be observed at a higher piperine concentration $(40 \mu \mathrm{g} / \mathrm{ml})$ at a low INH concentration of $0.0325 \mu \mathrm{g} / \mathrm{ml}$ (Figure 5B). The results indicate that the higher level of INH or PZA resistance in the Rv1258c S292L mutant is caused by higher efflux activity of the S292L mutant protein that could be inhibited by piperine.

\section{PZA-Resistant Rv1258c S292L Mutant Accumulates Less Drug POA}

It has been shown that $\left[{ }^{14} \mathrm{C}\right] \mathrm{PZA}$ is converted to $\left[{ }^{14} \mathrm{C}\right] \mathrm{POA}$ in M. tuberculosis and accumulates in the cell to some extent due to a weak efflux of POA (Zhang et al., 1999). To determine if Rv1258c V219A and S292L mutants, which are confirmed to be PZA-resistant in susceptibility testing (Figure 3), can pump $\left[{ }^{14} \mathrm{C}\right] \mathrm{PZA}$ out of the cell more effectively, we performed the $\left[{ }^{14} \mathrm{C}\right] \mathrm{PZA}$ uptake experiment comparing the amount of $\left[{ }^{14} \mathrm{C}\right] \mathrm{POA}$ accumulated in the cell in the Rv1258c mutants and that of the control parent strain. It was found that the intracellular concentration of POA in Rv1258c mutants was lower than that of PZA-susceptible M. tuberculosis H37Ra (Figure 6), indicating that PZA accumulated to a lesser extent in the PZA-resistant Rv1258c S292L and V219A mutants-which is an indication of higher efflux activity of the Rv1258c mutants-than the parent strain. In addition, the extent of $\left[{ }^{14} \mathrm{C}\right] \mathrm{POA}$ accumulation was in accordance with the degree of resistance of the Rv1258c mutants, with the S292L mutant accumulating less POA than the V219A

\section{REFERENCES}

Ainsa, J. A., Blokpoel, M. C., Otal, I., Young, D. B., De Smet, K. A., and Martin, C. (1998). Molecular cloning and characterization of Tap, a putative multidrug efflux pump present in Mycobacterium fortuitum and Mycobacterium tuberculosis. J. Bacteriol. 180, 5836-5843.

Andries, K., Villellas, C., Coeck, N., Thys, K., Gevers, T., Vranckx, L., et al. (2014). Acquired resistance of Mycobacterium tuberculosis to bedaquiline. PLoS One 9:e102135. doi: 10.1371/journal.pone.0102135

Banerjee, A., Dubnau, E., Quemard, A., Balasubramanian, V., Um, K. S., Wilson, T., et al. (1994). inhA, a gene encoding a target for isoniazid and ethionamide in Mycobacterium tuberculosis. Science 263, 227-230. doi: 10.1126/science. 8284673

Cheng, S. J., Thibert, L., Sanchez, T., Heifets, L., and Zhang, Y. (2000). pncA mutations as a major mechanism of pyrazinamide resistance in Mycobacterium tuberculosis: spread of a monoresistant strain in Quebec. Can. Antimicrob. Agents Chemother. 44, 528-532. doi: 10.1128/AAC.44.3.528-532.2000

Cloete, R., Kapp, E., Joubert, J., Christoffels, A., and Malan, S. F. (2018). Molecular modelling and simulation studies of the Mycobacterium tuberculosis multidrug efflux pump protein Rv1258c. PLoS One 13:e0207605. doi: 10.1371/journal. pone.0207605

Hartkoorn, R. C., Uplekar, S., and Cole, S. T. (2014). Cross-resistance between clofazimine and bedaquiline through upregulation of MmpL5 in Mycobacterium tuberculosis. Antimicrob. Agents Chemother. 58, 2979-2981. doi: 10.1128/AAC.00037-14

Heinrichs, M. T., May, R. J., Heider, F., Reimers, T., Sy, S. K., Peloquin, C. A., et al. (2018). Mycobacterium tuberculosis strains H37Ra and H37Rv have equivalent minimum inhibitory concentrations to most antituberculosis drugs. Int. J. Mycobacteriol. 7, 156-161. doi: 10.4103/ijmy.ijmy_33_18

Jiang, X., Zhang, W., Zhang, Y., Gao, F., Lu, C., Zhang, X., et al. (2008). Assessment of efflux pump gene expression in a clinical isolate Mycobacterium tuberculosis mutant, while both mutants accumulated less POA than the parent strain (Figure 6B).

In conclusion, we demonstrate that point mutations in efflux pump Rv1258c found in clinical isolates can play an important role in conferring clinically relevant drug resistance to multiple drugs including PZA, SM and INH. Our findings could explain some previously unaccounted drug resistance in drug-resistant clinical strains and indicate efflux pump mutations may need to be taken into consideration for improved molecular detection of drug resistance in $M$. tuberculosis. Furthermore, efflux pump inhibitor piperine may be used as adjunct for more effective possible treatment of multi-drug resistant $M$. tuberculosis in future studies.

\section{AUTHOR CONTRIBUTIONS}

$\mathrm{YZ}$, JL, XH, and VD designed the experiments. JL, WS, SZ, DM, $\mathrm{KS}$, and $\mathrm{OB}$ performed the experiments. JL, WS, XH, VD, and YZ analyzed the data. JL and $\mathrm{YZ}$ wrote the manuscript.

\section{FUNDING}

This work was supported by the United States-Russia (NIHRFBR) Collaborative Research Partnership on the Prevention and Treatment of HIV/AIDS and Comorbidities (AI108535) and AI099512, and the Russian Foundation for Basic Research (RFBR) grant 13-04-91444.

by real-time reverse transcription PCR. Microb. Drug Resist. 14, 7-11. doi: 10.1089/mdr.2008.0772

Kanji, A., Hasan, R., Ali, A., Zaver, A., Zhang, Y., and Imtiaz, K. (2017). Single nucleotide polymorphisms in efflux pumps genes in extensively drug resistant Mycobacterium tuberculosis isolates from Pakistan. Tuberculosis 107, 20-30. doi: 10.1016/j.tube.2017.07.012

Kardan-Yamchi, J., Kazemian, H., Haeili, M., Harati, A. A., Amini, S., and Feizabadi, M. M. (2019). Expression analysis of 10 efflux pump genes in multi/extensively drug-resistant Mycobacterium tuberculosis isolates of clinical origin. J. Glob. Antimicrob. Resist. doi: 10.1016/j.jgar.2019.01.003 [Epub ahead of print].

Li, X. Z., and Nikaido, H. (2009). Efflux-mediated drug resistance in bacteria: an update. Drugs 69, 1555-1623.

Liu, J., Takiff, H. E., and Nikaido, H. (1996). Active efflux of fluoroquinolones in Mycobacterium smegmatis mediated by LfrA, a multidrug efflux pump. J. Bacteriol. 178, 3791-3795. doi: 10.1128/jb.178.13.3791-3795. 1996

Parish, T., and Stoker, N. G. (2000). Use of a flexible cassette method to generate a double unmarked Mycobacterium tuberculosis tlyA plcABC mutant by gene replacement. Microbiology 146, 1969-1975. doi: 10.1099/00221287-146-8- 1969

Ramon-Garcia, S., Martin, C., Thompson, C. J., and Ainsa, J. A. (2009). Role of the Mycobacterium tuberculosis P55 efflux pump in intrinsic drug resistance, oxidative stress responses, and growth. Antimicrob. Agents Chemother. 53, 3675-3682. doi: 10.1128/AAC.00550-09

Ramon-Garcia, S., Mick, V., Dainese, E., Martin, C., Thompson, C. J., De Rossi, E., et al. (2012). Functional and genetic characterization of the tap efflux pump in Mycobacterium bovis BCG. Antimicrob. Agents Chemother. 56, 2074-2083. doi: 10.1128/AAC.05946-11

Sharma, S., Kumar, M., Nargotra, A., Koul, S., and Khan, I. A. (2010). Piperine as an inhibitor of Rv1258c, a putative multidrug efflux pump of Mycobacterium tuberculosis. J. Antimicrob. Chemother. 65, 1694-1701. doi: 10.1093/jac/dkq186 
Shi, W., Zhang, X., Jiang, X., Yuan, H., Lee, J. S., Barry, C. E. III, et al. (2011). Pyrazinamide inhibits trans-translation in Mycobacterium tuberculosis. Science 333, 1630-1632. doi: 10.1126/science.1208813

Shur, K. V., Maslov, D. A., Mikheecheva, N. E., Akimov, N. I., Bekker, O. B., and Danilenko, V. N. (2017). The Intrinsic antibiotic resistance to $\beta$-Lactams, macrolides, and fluoroquinolones of mycobacteria Is mediated by the whiB7 and tap Genes. Russ. J. Genet. 53, 1006-1015. doi: 10.1134/S10227954170 80087

WHO (2018). Global Tuberculosis Report. Available at: http://www.who.int/tb/ publications/global_report/en/

Yee, M., Gopal, P., and Dick, T. (2017). Missense mutations in the unfoldase $\mathrm{ClpC} 1$ of the caseinolytic protease complex are associated with pyrazinamide resistance in Mycobacterium tuberculosis. Antimicrob. Agents Chemother. 61:e02342-16. doi: 10.1128/AAC.02342-16

Zhang, S., Chen, J., Shi, W., Cui, P., Zhang, J., Cho, S., et al. (2017). Mutation in clpC1 encoding an ATP-dependent ATPase involved in protein degradation is associated with pyrazinamide resistance in Mycobacterium tuberculosis. Emerg. Microbes Infect. 6:e8. doi: 10.1038/emi.2017.1

Zhang, S., Chen, J., Shi, W., Liu, W., Zhang, W. H., and Zhang, Y. (2013). Mutations in panD encoding aspartate decarboxylase are associated with pyrazinamide resistance in Mycobacterium tuberculosis. Emerg. Microbes Infect. 2:e34. doi: 10.1038/emi.2013.38

Zhang, Y., Heym, B., Allen, B., Young, D., and Cole, S. (1992). The catalaseperoxidase gene and isoniazid resistance of Mycobacterium tuberculosis. Nature 358, 591-593. doi: 10.1038/358591a0

Zhang, Y., and Mitchison, D. (2003). The curious characteristics of pyrazinamide: a review. Int. J. Tuberc. Lung Dis. 7, 6-21.
Zhang, Y., Scorpio, A., Nikaido, H., and Sun, Z. (1999). Role of acid pH and deficient efflux of pyrazinoic acid in unique susceptibility of Mycobacterium tuberculosis to pyrazinamide. J. Bacteriol. 181, 2044-2049.

Zhang, Y., Shi, W., Zhang, W., and Mitchison, D. (2013). Mechanisms of pyrazinamide action and resistance. Microbiol. Spectr. 2, 1-12. doi: 10.1128/ microbiolspec.MGM2-0023-2013

Zhang, Y., and Yew, W. W. (2009). Mechanisms of drug resistance in Mycobacterium tuberculosis. Int. J. Tuberc. Lung Dis. 13, 1320-1330.

Zhang, Y., Zhang, J., Cui, P., Zhang, Y., and Zhang, W. (2017). Identification of novel efflux proteins Rv0191, Rv3756c, Rv3008, and Rv1667c involved in pyrazinamide resistance in Mycobacterium tuberculosis. Antimicrob. Agents Chemother. 61, e940-e917. doi: 10.1128/AAC.00940-17

Zhu, Y. H., Gao, Y. F., Chen, F., Liu, W., Zhai, M. X., Zhai, W. J., et al. (2011). Identification of novel $\mathrm{T}$ cell epitopes from efflux pumps of Mycobacterium tuberculosis. Immunol. Lett. 140, 68-73. doi: 10.1016/j.imlet.2011.06.009

Conflict of Interest Statement: The authors declare that the research was conducted in the absence of any commercial or financial relationships that could be construed as a potential conflict of interest.

Copyright (c) 2019 Liu, Shi, Zhang, Hao, Maslov, Shur, Bekker, Danilenko and Zhang. This is an open-access article distributed under the terms of the Creative Commons Attribution License (CC BY). The use, distribution or reproduction in other forums is permitted, provided the original author(s) and the copyright owner(s) are credited and that the original publication in this journal is cited, in accordance with accepted academic practice. No use, distribution or reproduction is permitted which does not comply with these terms. 